\title{
Oral immunoglobulin levels are not a good surrogate for cervical immunoglobulin levels
}

\section{Troy J. Kemp ${ }^{1}$, Mahboobeh Safaeian ${ }^{2}$, Samantha Miner ${ }^{1}$, Marcus C. Williams ${ }^{1}$, Ana Cecilia Rodriguez ${ }^{3}$, Rolando Herrero ${ }^{3,4}$, Allan Hildesheim ${ }^{2}$ and Ligia A. Pinto ${ }^{1}$ *}

\author{
${ }^{1}$ HPV Immunology Laboratory, SAIC-Frederick, Inc., NCl-Frederick, Frederick, MD, USA \\ 2 Division of Cancer Epidemiology and Genetics, National Cancer Institute, National Institutes of Health, Bethesda, MD, USA \\ ${ }_{3}$ Proyecto Epidemiológico Guanacaste, Fundación INCIENSA, San Jose, Costa Rica \\ ${ }^{4}$ Prevention and Implementation Group, International Agency for Research on Cancer, Lyon, France
}

\section{Edited by:}

Karina Braga Ribeiro, Hospital A C

Camargo, Brazil

\section{Reviewed by:}

Min Dai, Chinese Academy of

Medical Sciences, China

Shu-Chun Chuang, Imperial College

London, UK

${ }^{*}$ Correspondence:

Ligia A. Pinto, HPV Immunology Laboratory, SAIC-Frederick, Inc., NCl-Frederick, Building 469, Room 111, Frederick, MD 21702, USA e-mail: pintol@mail.nih.gov

\begin{abstract}
Background: We sought to determine whether oral secretions could be used as a surrogate for cervical secretions for monitoring cervical immunoglobulin (lg) levels. To do so, we examined (1) whether oral IgG and IgA levels correlated with those observed at the cervix, and (2) whether time of menstrual cycle and other factors previously reported to influence cervical Ig levels were associated with oral IgG and IgA levels. Methods: We obtained oral samples from a group of 85 Costa Rican woman 25-35 years of age measured at three time points during one menstrual cycle. Total $\lg G$ and $\lg A$ levels were measured by ELISA. Generalized estimating equations methods that account for repeated measures were used to evaluate the association between oral and cervical lg levels and to evaluate the association between various covariates and oral IgA and IgG levels. Results: We did not observe an association between oral and cervical IgG [linear regression coefficient (LRC) $0.01 ; 95 \%$ $\mathrm{Cl},-0.05$ to 0.07 ] and IgA levels (LRC $0.02 ; 95 \% \mathrm{Cl},-0.04$ to 0.08 ). Oral IgG and IgA levels were not influenced by phase of menstrual cycle, in contrast to what has previously been observed for cervical Ig levels. Conclusion: Our data suggest that oral IgG and IgA measures are not a good surrogate for cervical IgG and IgA levels. Future studies should examine whether antigen-specific antibody responses induced by vaccination correlate across mucosal sites.
\end{abstract}

Keywords: oral fluid, IgG, IgA, cervical secretion, menstrual cycle

\section{INTRODUCTION}

During the past decade, oral sampling has gained increased attention as an alternative to plasma in monitoring immunity in epidemiological studies. Oral sampling has a number of advantages when compared to venipuncture, including the fact that sampling is simple, non-invasive, painless, and allows for self-collection (Wade and Haegele, 1991; Kirschbaum and Hellhammer, 1994). Many biomarkers have been evaluated in oral secretions including hormones (Riad-Fahmy et al., 1982; Wade and Haegele, 1991), vaccine-induced antibodies (Kozlowski et al., 1999; RowhaniRahbar et al., 2009), and cytokines (Streckfus et al., 2002). This makes oral secretions a relevant source for monitoring hormone levels and antibody responses in vaccinated individuals. We were interested in determining whether oral immunoglobulin (Ig) levels represent a good surrogate for cervical mucosal Ig levels. This is of particular relevance for monitoring recipients of the recently licensed prophylactic HPV vaccines (Muñoz et al., 2009; Paavonen et al., 2009). The new HPV vaccines induce a strong antibody response that is believed to be responsible for the protection against vaccine-related type infections. Because vaccination is administered systemically whereas protection occurs locally at the genital tract mucosa, we have had an interest in better understanding the patterns of both systemic and local immune responses and their interrelationship.
We and others have shown that Ig levels in cervical secretions may be hormonally influenced, as reflected by fluctuations in cervical IgG and IgA during the menstrual cycle, with levels being lowest around ovulation and higher in the follicular and luteal phases of the cycle (Nardelli-Haefliger et al., 2003; Safaeian et al., 2009a). In contrast, systemic Ig levels have been shown not to be affected by the menstrual cycle and do not correlate with cervical Ig levels (Safaeian et al., 2009b).

It is still unknown whether oral levels of Ig reflect those at the cervix. To determine whether oral secretions can be used as an alternative to cervical secretions to monitor local Ig levels, we examined (1) the association between oral and cervical Ig (both IgG and IgA) levels, and (2) the determinants of total oral IgG and IgA levels to see whether they are similar to those observed at the cervix.

\section{MATERIALS AND METHODS POPULATION}

To evaluate mucosal and systemic levels of total IgG and IgA over the menstrual cycle, a subset of young adult women from Costa Rica who were participants in a NCI-sponsored study designed to examine the natural history of HPV infection and cervical intraepithelial neoplasia (Herrero et al., 1997) were contacted if they were 25-35 years old, had no evidence of high-grade cervical lesions, 
were not pregnant, and had an intact uterus. The details of this study have been described previously (Safaeian et al., 2009a). In brief, a total of 202 women were invited to participate and asked to agree to an interview and the collection of cervical and oral secretions (blood was also collected and results reported previously; Safaeian et al., 2009b) at three time points during one menstrual cycle within the following time frames: visit 1 (follicular phase) 5-8 days after cycle onset, visit 2 (periovulatory phase) 14-16 days after cycle onset, and visit 3 (luteal phase) 19-22 days after cycle onset. After excluding women who were pregnant $(n=3)$, currently breast-feeding $(n=17)$, or had menstrual cycles that were either too short ( $<25$ days) or too long ( $>36$ days; $n=28$ ), we selected for study a subset of 104 women, 20 of whom were oral contraceptive users (the remaining were naturally cycling). For the present analysis, we further excluded women who did not provide specimens at all three visits $(n=1)$, whose oral sponge weight was unusually low (lower than the mean weight of dry sponges; $n=4$ ), whose cervical sponge weight was unusually low $(n=13)$, and whose cervical IgG and IgA levels were considered to be outliers $(n=1)$. A total of 85 women were included in the final analysis. All participants signed an informed consent, and the study was approved by the ethical committees at the National Cancer Institute, USA and INCIENSA, Costa Rica.

\section{SAMPLE COLLECTION AND EXTRACTION}

Oral samples were collected using the cellulose-based Ultracell sponges by holding a sponge on the inner cheek for $15 \mathrm{~s}$ and turning it over to collect oral secretions for another $15 \mathrm{~s}$ on the opposite cheek. Collection of cervical secretions has been described previously (Kemp et al., 2008; Safaeian et al., 2009a). Following collection, the sponges (both oral and cervical) were immediately stored in liquid nitrogen. The secretions were extracted from Ultracell sponges according to the following protocol. Three hundred microliter of extraction buffer (PBS, Invitrogen, Grand Island, NY, USA; 1.5\% NaCl, Sigma-Aldrich, St. Louis, MO, USA; $100 \mu \mathrm{g} / \mathrm{ml}$ Aprotinin, Sigma-Aldrich; $150 \mathrm{ng} / \mathrm{ml}$ mouse IgG1, extraction control, BD Biosciences, San Jose, CA, USA) was added to the top of each sponge. The sponges were incubated at $4^{\circ} \mathrm{C}$ for $30 \mathrm{~min}$ prior to centrifugation at $13,000 \times g$ for $15 \mathrm{~min}$ at $4^{\circ} \mathrm{C}$. An additional $300 \mu \mathrm{l}$ of extraction buffer (sans extraction control) was added to each sponge and immediately centrifuged. Prior to adding $4 \mu \mathrm{l}$ of fetal calf serum for storage, $20 \mu \mathrm{l}$ of extract was saved for protein analysis, and $5 \mu \mathrm{l}$ was tested for presence of hemoglobin (Hemastix, Bayer, Elkhart, IN, USA).

\section{DETERMINATION OF IMMUNOGLOBULIN LEVELS}

Total human IgG, human IgA, and extraction control (mouse IgG1) were measured in duplicate using an ELISA according to the manufacturer's protocol (Bethyl Laboratories, Montgomery, TX, USA). In order to account for variations in the amount of oral secretion collected between participants, the antibody levels were standardized based on the following formula: [specimen weight (g) - mean dry sponge weight $(\mathrm{g})+0.6(\mathrm{~g})] /[$ specimen weight (g) - mean dry sponge weight]; 0.6 (g) refers to the weight of the extraction buffer added to each specimen. Furthermore, we added a standardized amount of mouse IgG1 to the extraction buffer in order to monitor extraction efficiency. The median percent recovery was 75.5\% (IQR: 62.7-87.1\%). Results from analyses that evaluated standardized oral Ig levels corrected for percent recovery did not differ from those obtained using the uncorrected standardized oral Ig levels except for the correlation between oral IgA levels amongst smoking status [linear regression coefficient (LRC) 0.47 ; 95\% CI (0.02-0.92); $p=0.04$; data not shown].

\section{STATISTICAL ANALYSES}

The IgG and IgA levels were normalized by log transformation. Geometric mean titers (GMT) of oral IgG and IgA were computed and reported by categories of the covariates examined. Our main objective was to examine the association of oral IgG and IgA levels with cervical IgG and IgA, respectively. Other variables considered as potential confounders were: menstrual cycle phase (follicular, periovulatory, and luteal), oral contraceptive use (current versus not), age (25-29, 30-32, and 33-35 years), smoking (current versus not), cervical HPV infection (current versus not), acute illness (current versus not), parity $(0,1-3$, and $\geq 4)$, saliva sponge weight $(<0.21$ versus $>0.21 \mathrm{~g})$, and presence of hemoglobin (negative, trace, and positive). The Kruskal-Wallis test was used to assess significant changes in oral IgG and IgA levels during the three menstrual cycle phases. Because we had three observations for each participant, we applied generalized estimating equations (GEE) models for correlated data to determine which factors were associated with oral IgG and IgA levels. Robust methods for linear regression with unstructured correlation structure were used to estimate standard errors and coefficients adjusted for multiple observations for each participant. A $p<0.05$ is considered significant. LRC and associated $95 \%$ confidence intervals (CI) are presented. Analysis was performed with STATA 9.2 (StataCorp LP, College Station, TX, USA), SAS 9.3, and JMP 7 statistical programs (SAS Institute Inc., Cary, NC, USA).

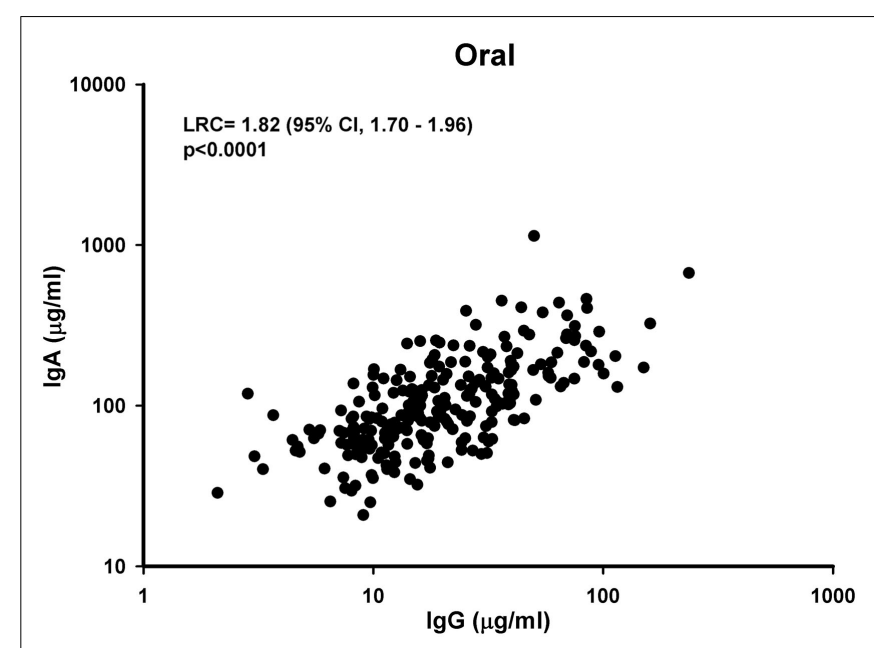

FIGURE 1 |Association of oral IgG to oral IgA levels. Oral samples collected from the three phases of the menstrual cycle from 85 participants were measured for $\lg G$ and $\lg A$ levels. The graph represents a scatter plot of untransformed levels of oral IgG and IgA from all three phases of the menstrual cycle from each participant, and a $p$-value $<0.05$ was considered significant. LRC, Linear Regression Coefficient. 


\section{RESULTS}

A total of 85 women were included in this analysis. Each woman was sampled at three time points during a single menstrual cycle for a total of 255 oral samples with matching cervical samples. The overall geometric mean concentration of oral IgG and IgA from the 255 samples was 19.6 and $101 \mu \mathrm{g} / \mathrm{ml}$, respectively. The levels of total IgG in oral samples were 30 -fold lower than the IgG levels found in the cervix (GMT for cervical IgG $=567 \mu \mathrm{g} / \mathrm{ml}$ ), and the levels of IgA in oral samples were 2.5-fold lower than the levels of IgA detected in the cervix (GMT for cervical IgA $=269 \mu \mathrm{g} / \mathrm{ml}$ ).

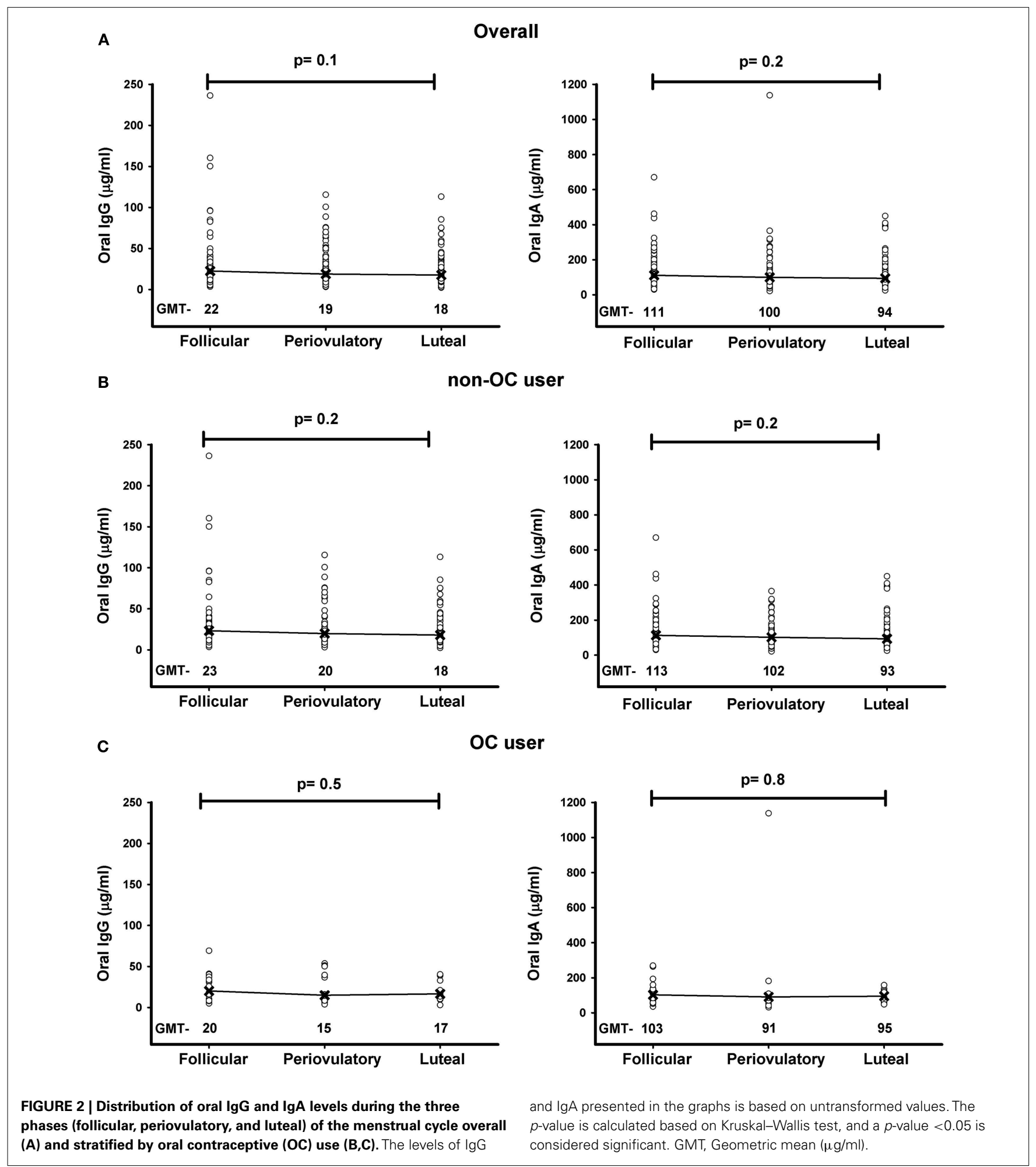


Oral IgG levels were significantly associated with oral IgA levels from the 255 samples considered as a whole (LRC: 1.82; 95\% CI, 1.70-1.96; Figure 1).

Because, we previously reported that cervical levels of IgG and IgA were significantly lower during the periovulatory phase of the menstrual cycle among naturally cycling women (Safaeian et al., 2009a), we examined whether the menstrual cycle affected oral IgG and IgA levels. In contrast to what we reported previously at the cervix, we observed no significant change in oral levels of $\operatorname{IgG}(p=0.1)$ or $\operatorname{IgA}(p=0.2)$ between phase of menstrual cycle (Figure 2). This was true overall (Figure 2A) and in analysis stratified by oral contraceptive use (Figures 2B,C).

Figure 3A depicts the association between oral IgG and cervical IgG from the 255 samples considered as a whole regardless of the phase of the menstrual cycle. Oral IgG levels were not associated with cervical IgG levels (LRC: $0.01 ; 95 \% \mathrm{CI},-0.05$ to 0.07 ). Similarly as depicted in Figure 3B, oral IgA levels were not associated with cervical IgA levels (LRC: $0.02 ; 95 \%$ CI, -0.04 to 0.08 ).

When other potential determinants of oral IgG and IgA levels were evaluated (Table 1), only sponge weight was significantly associated with oral IgG (LCR: $-0.72,95 \% \mathrm{CI},-0.86$ to -0.59 ) and oral IgA levels (LCR: $-0.54 ; 95 \% \mathrm{CI},-0.66$ to -0.42 ). Although not statistically significant, we did observe that oral IgG and IgA levels were elevated in specimens which hemoglobin was detectable by our Hemastix strip test (Table 1).

\section{DISCUSSION}

Saliva represents a potential alternative sample collection method for immune monitoring studies. This could simplify efforts to monitor the long-term immunological impact of vaccines such as the recently approved prophylactic HPV vaccines. However, it is still unknown whether saliva levels of antibodies reflect local levels at the cervix. In this study, we evaluated whether total oral Ig measures were good proxies for genital tract Ig levels. The aims were to (1) directly compare Ig levels measured in the oral cavity against those measured at the cervix and (2) examine whether factors previously found to affect levels of Ig at the cervix were also associated with oral Ig levels.
We showed that IgG and IgA in oral secretions are lower than levels found in the cervix and not reflective of IgG or IgA levels in the cervix. The overall levels of oral IgG and IgA reported here are in agreement with other studies (Norhagen et al., 1989; Tamashiro and Constantine, 1994; Engstrom et al., 1996; Kozlowski et al., 1999), and the levels of oral IgG and IgA suggest that the collection device in our study absorbed whole saliva, which consists of all oral mucosa products. Furthermore, these results suggest that our collection and storage method preserved immunoglobulins even though oral fluid contains enzymes capable of degrading proteins such as immunoglobulins.

We examined the association of oral IgG and IgA with menstrual cycle and hormonal contraceptive use. Oral IgG and IgA levels were not affected by phase of menstrual cycle or by oral contraceptive use. These observations, while consistent with previously reported findings for plasma levels (Safaeian et al., 2009b), contrast with the reported strong effect of menstrual cycle and oral contraceptive use on cervical Ig levels (Safaeian et al., 2009a), further reinforcing the need for caution when using oral Ig measurements to reflect levels at the cervix. Although our study did not directly measure antigen-specific vaccine-induced antibody levels, our results suggest caution in using oral specimens to monitor long-term impact of vaccination programs such as the recently approved HPV vaccine.

The only statistically significant correlate of oral IgG and IgA we identified was oral sponge collection weight. The increased IgG and IgA levels among lighter weight sponges suggests that the calculated dilution factor may artificially increase the concentration of oral IgG and IgA when low levels of fluid are obtained.

Previous studies have shown that there was modest to high correlation between cervical and plasma anti-HPV16 levels (NardelliHaefliger et al., 2003; Kemp et al., 2008); however, this was shown to be the case among individuals who received the HPV vaccine only when menstrual cycle fluctuations were controlled by oral contraceptive use (Nardelli-Haefliger et al., 2003). Although antigen-specific responses have been reported to be detected in oral fluid from vaccine recipients (Rowhani-Rahbar et al., 2009), it is still unclear whether such responses would correlate with levels observed at the cervix among naturally cycling women.

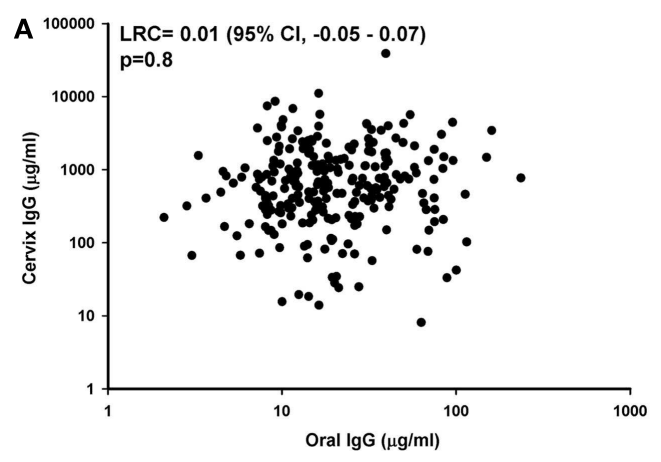

FIGURE 3 | Comparison of IgG and IgA levels between oral and cervical specimens. Matching oral and cervical secretion samples from the three phases of the menstrual cycle from 85 participants were measured for $\lg G$ and $\lg A$ levels. The scatter plots of oral-cervix $\lg G(\mathbf{A})$

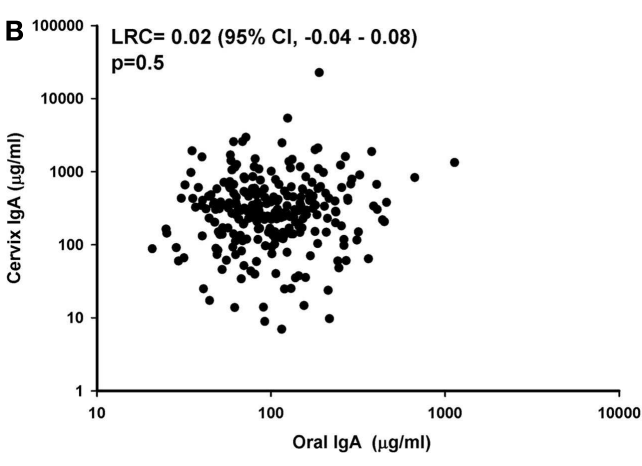

and $\lg A$ (B) levels are plotted based on untransformed values and include the three phases of the menstrual cycle from each participant. A $p$-value $<0.05$ was considered significant, and LRC stands for Linear Regression Coefficient. 
Table 1 | Association between covariates of interest and oral $\lg G$ and $\lg A(\mu \mathrm{g} / \mathrm{ml})$ levels.

\begin{tabular}{|c|c|c|c|c|c|}
\hline & $\mathbf{N}$ & $\begin{array}{l}\lg G(\mu \mathrm{g} / \mathrm{ml}) \\
\mathrm{GMT}^{\mathrm{a}}\end{array}$ & $\begin{array}{l}\operatorname{lgG} \\
\operatorname{LRC}(95 \% \mathrm{Cl})^{b}\end{array}$ & $\begin{array}{l}\lg A(\mu \mathrm{g} / \mathrm{ml}) \\
\mathrm{GMT}^{\mathrm{a}}\end{array}$ & $\begin{array}{l}\lg A \\
\operatorname{LRC}(95 \% \mathrm{Cl})^{b}\end{array}$ \\
\hline Oral lgd & 255 & 19.6 & & 101.3 & \\
\hline Cervix $\lg ^{\mathrm{d}}$ & 255 & 566.7 & $0.01(-0.05$ to 0.07$)$ & 268.6 & $0.02(-0.04$ to 0.08$)$ \\
\hline Plasma lg & 255 & 8204.5 & $0.74(0.12$ to 1.35$)$ & 2030.1 & $-0.03(-0.32$ to 0.25$)$ \\
\hline \multicolumn{6}{|l|}{ AGE } \\
\hline $27-30$ & 84 & 22 & $-0.05(-0.11 \text { to } 0.01)^{c}$ & 98.5 & $0.01(-0.04 \text { to } 0.06)^{c}$ \\
\hline $31-33$ & 96 & 19.4 & & 101.0 & \\
\hline $34-35$ & 75 & 17.4 & & 105.0 & \\
\hline \multicolumn{6}{|c|}{ ORAL COLLECTION SPONGE WEIGHT } \\
\hline$>0.21 \mathrm{~g}$ & 130 & 12.9 & Reference & 74.9 & Reference \\
\hline$<0.21 \mathrm{~g}$ & 125 & 30.1 & $-0.72(-0.86$ to -0.59$)$ & 138.6 & $-0.54(-0.66$ to -0.42$)$ \\
\hline \multicolumn{6}{|c|}{ HEMOGLOBIN IN ORAL SECRETIONS } \\
\hline Negative & 21 & 17.2 & Reference & 95.6 & Reference \\
\hline Trace & 184 & 17.3 & $-0.09(-0.44$ to 0.26$)$ & 97.2 & $-0.04(-0.34$ to 0.26$)$ \\
\hline Positive & 50 & 32.2 & $0.23(-0.17$ to 0.62$)$ & 120.7 & $0.10(-0.23$ to 0.43$)$ \\
\hline \multicolumn{6}{|c|}{ CURRENT OC USE } \\
\hline No & 191 & 19.3 & Reference & 98.6 & Reference \\
\hline Yes & 44 & 18.0 & $0.01(-0.31$ to 0.34$)$ & 104.2 & $0.06(-0.2$ to 0.32$)$ \\
\hline \multicolumn{6}{|c|}{ HPV INFECTION } \\
\hline No & 207 & 19.5 & Reference & 102.1 & Reference \\
\hline Yes & 42 & 18.5 & $-0.05(-0.44$ to 0.34$)$ & 95.1 & $-0.08(-0.38$ to 0.23$)$ \\
\hline \multicolumn{6}{|l|}{ PARITY } \\
\hline 0 & 30 & 19.8 & Reference & 124.4 & Reference \\
\hline $1-3$ & 162 & 17.5 & $-0.12(-0.57$ to 0.33$)$ & 91.6 & $-0.30(-0.65$ to 0.04$)$ \\
\hline$\geq 4$ & 63 & 25.7 & $0.27(-0.23$ to 0.77$)$ & 119.1 & $-0.04(-0.43$ to 0.35$)$ \\
\hline \multicolumn{6}{|c|}{ CURRENT ILLNESS } \\
\hline No & 174 & 19.9 & Reference & 101.6 & Reference \\
\hline Yes & 81 & 18.9 & $-0.02(-0.19$ to 0.15$)$ & 100.6 & $0.02(-0.13$ to 0.16$)$ \\
\hline \multicolumn{6}{|c|}{ CURRENT SMOKER } \\
\hline No & 228 & 19.1 & Reference & 97.6 & Reference \\
\hline Yes & 27 & 23.5 & $0.22(-0.25$ to 0.69$)$ & 139.1 & $0.35(-0.01$ to 0.72$)$ \\
\hline
\end{tabular}

${ }^{a}$ Geometric mean values of $\lg G$ and $\lg A$ are based on log transformed values which have been transformed back to the original measurements.

${ }^{b}$ The Generalized Estimation Equation (GEE) model was used to evaluate the associations between the listed covariates and log transformed oral lgG and lgA values. The Linear Regression Coefficient (LRC) values are presented along with $95 \% \mathrm{Cls}$

${ }^{c}$ Age entered as a continuous variable in the model.

${ }^{d}$ The immunoglobulin measures presented reflect the geometric mean of all samples regardless of the menstrual cycle.

Bold text represents an $L R C$ being significant at $p<0.05$.

In conclusion, our data suggest that oral IgG and IgA are not a good surrogate for total IgG and IgA measures in cervical secretions. Future studies will need to assess whether antigen-specific antibody responses in oral samples obtained from vaccinees may better reflect levels observed in cervix before such markers can be used as an alternative to cervical secretion measures when monitoring local antigen-specific humoral responses.

\section{REFERENCES}

Engstrom, P. E., Norhagen, G., Osipova, L., Helal, A., Wiebe, V., Brusco, A., Carbonara, A. O., Lefranc, G., and Lefranc, M. P. (1996). Salivary IgG subclasses in individuals with and without homozygous IGHG gene deletions. Immunology 89, 178-182. C., Hildesheim, A., Balmaceda, I., Sherman, M. E., Greenberg, M., Cardenas, F., Gomez, V., Helgesen, K., Morales, J., Hutchinson, M.,
Herrero, R., Schiffman, M. H., Bratti,

\section{ACKNOWLEDGMENTS}

This project has been funded in whole or in part with federal funds from the National Cancer Institute, National Institutes of Health, under Contract No. HHSN261200800001E. The content of this publication does not necessarily reflect the views or policies of the Department of Health and Human Services, nor does mention of trade names, commercial products, or organizations imply endorsement by the U.S. Government.

Mango, L., Alfaro, M., Potischman, N. W., Wacholder, S., Swanson, C., and Brinton, L. A. (1997). Design and methods of a populationbased natural history study of cervical neoplasia in a rural province of Costa Rica: the Guanacaste
Project. Rev. Panam. Salud Publica 1, 362-375.

Kemp, T. J., Garcia-Pineres, A., Falk, R. T., Poncelet, S., Dessy, F., Giannini, S. L., Rodriguez, A. C., Porras, C., Herrero, R., Hildesheim, A., and Pinto, L. A. (2008). Evaluation of 
systemic and mucosal anti-HPV16 and anti-HPV18 antibody responses from vaccinated women. Vaccine 26 , 3608-3616.

Kirschbaum, C., and Hellhammer, D. H. (1994). Salivary cortisol in psychoneuroendocrine research: recent developments and applications. Psychoneuroendocrinology 19, 313-333.

Kozlowski, P. A., Cu-Uvin, S., Neutra, M. R., and Flanigan, T. P. (1999). Mucosal vaccination strategies for women. J. Infect. Dis. 179(Suppl. 3), S493-S498.

Muñoz, N., Manalastas, R. Jr., Pitisuttithum, P., Tresukosol, D., Monsonego, J., Ault, K., Clavel, C., Luna, J., Myers, E., Hood, S., Bautista, O., Bryan, J., Taddeo, F. J., Esser, M. T., Vuocolo, S., Haupt, R. M., Barr, E., and Saah, A. (2009). Safety, immunogenicity, and efficacy of quadrivalent human papillomavirus (types 6, 11, 16, 18) recombinant vaccine in women aged $24-45$ years: a randomised, double-blind trial. Lancet 373, 1949-1957.

Nardelli-Haefliger, D., Wirthner, D., Schiller, J. T., Lowy, D. R., Hildesheim, A., Ponci, F, and De Grandi, P. (2003). Specific antibody levels at the cervix during the menstrual cycle of women vaccinated with human papillomavirus 16 virus-like particles. J. Natl. Cancer Inst. 95, 1128-1137.

Norhagen, G., Engstrom, P. E., Hammarstrom, L., Soder, P. O., and Smith, C. I. (1989). Immunoglobulin levels in saliva in individuals with selective IgA deficiency: compensatory IgM secretion and its correlation with HLA and susceptibility to infections. J. Clin. Immunol. 9, 279-286.

Paavonen, J., Naud, P., Salmerón, J., Wheeler, C. M., Chow, S. N., Apter, D., Kitchener, H., Castellsague, X., Teixeira, J. C., Skinner, S. R., Hedrick, J., Jaisamrarn, U., Limson, G., Garland, S., Szarewski, A., Romanowski, B., Aoki, F. Y., Schwarz, T. F., Poppe, W. A. J., Bosch, F. X., Jenkins, D., Hardt, K., Zahaf, T., Descamps, D., Struyf, F., Lehtinen, M., and Dubin, G. (2009). Efficacy of human papillomavirus (HPV)-16/18 AS04adjuvanted vaccine against cervical infection and precancer caused by oncogenic HPV types (PATRICIA): final analysis of a double-blind, randomised study in young women. Lancet 374, 301-314.

Riad-Fahmy, D., Read, G. F., Walker, R. F., and Griffiths, K. (1982). Steroids in saliva for assessing endocrine function. Endocr. Rev. 3, 367-395.

Rowhani-Rahbar, A., Carter, J. J., Hawes, S. E., Hughes, J. P., Weiss, N.
S., Galloway, D. A., and Koutsky, L. A. (2009). Antibody responses in oral fluid after administration of prophylactic human papillomavirus vaccines. J. Infect. Dis. 200 1452-1455.

Safaeian, M., Falk, R. T., Rodriguez, A. C., Hildesheim, A., Kemp, T., Williams, M., Morera, L., Barrantes, M., Herrero, R., Porras, C., and Pinto, L. (2009a). Factors associated with fluctuations in IgA and IgG levels at the cervix during the menstrual cycle. J. Infect. Dis. 199, 455-463.

Safaeian, M., Kemp, T., Falk, R. T. Rodriguez, A. C., Hildesheim, A., Williams, M., Porras, C., Herrero, R., and Pinto, L. A. (2009b). Determinants and correlation of systemic and cervical concentrations of total IgA and IgG. Cancer Epidemiol. Biomarkers Prev. 18, 2672-2676.

Streckfus, C., Bigler, L., and O'Bryan, T. (2002). Aging and salivary cytokine concentrations as predictors of whole saliva flow rates among women: a preliminary study. Gerontology 48, 282-288.

Tamashiro, H., and Constantine, N. T. (1994). Serological diagnosis of HIV infection using oral fluid samples. Bull. World Health Organ. 72, 135-143.

Wade, S. E., and Haegele, A. D. (1991). Time-integrated measurement of corticosteroids in saliva by oral diffusion sink technology. Clin. Chem. 37, 1166-1172.

Conflict of Interest Statement: The authors declare that the research was conducted in the absence of any commercial or financial relationships that could be construed as a potential conflict of interest.

Received: 28 March 2012; accepted: 30 May 2012; published online: 25 June 2012.

Citation: Kemp TJ, Safaeian M, Miner $S$, Williams MC, Rodriguez AC, Herrero $R$, Hildesheim A and Pinto LA (2012) Oral immunoglobulin levels are not a good surrogate for cervical immunoglobulin levels. Front. Oncol. 2:61. doi: 10.3389/fonc.2012.00061

This article was submitted to Frontiers in Cancer Epidemiology and Prevention, a specialty of Frontiers in Oncology. Copyright (c) 2012 Kemp, Safaeian, Miner, Williams, Rodriguez, Herrero, Hildesheim and Pinto. This is an openaccess article distributed under the terms of the Creative Commons Attribution Non Commercial License, which permits non-commercial use, distribution, and reproduction in other forums, provided the original authors and source are credited. 